\title{
Heralded Quantum Gates with Integrated Error Detection in Optical Cavities
}

\author{
J. Borregaard, ${ }^{1}$ P. Kómár, ${ }^{2}$ E. M. Kessler, ${ }^{2,3}$ A. S. Sørensen, ${ }^{1}$ and M. D. Lukin ${ }^{2}$ \\ ${ }^{1}$ The Niels Bohr Institute, University of Copenhagen, Blegdamsvej 17, DK-2100 Copenhagen Ø, Denmark \\ ${ }^{2}$ Department of Physics, Harvard University, Cambridge, Massachusetts 02138, USA \\ ${ }^{3}$ ITAMP, Harvard-Smithsonian Center for Astrophysics, Cambridge, Massachusetts 02138, USA
}

(Received 1 January 2015; published 17 March 2015)

\begin{abstract}
We propose and analyze heralded quantum gates between qubits in optical cavities. They employ an auxiliary qubit to report if a successful gate occurred. In this manner, the errors, which would have corrupted a deterministic gate, are converted into a nonunity probability of success: once successful, the gate has a much higher fidelity than a similar deterministic gate. Specifically, we describe that a heralded, near-deterministic controlled phase gate (CZ gate) with the conditional error arbitrarily close to zero and the success probability that approaches unity as the cooperativity of the system, $C$, becomes large. Furthermore, we describe an extension to near-deterministic $\mathrm{N}$-qubit Toffoli gate with a favorable error scaling. These gates can be directly employed in quantum repeater networks to facilitate near-ideal entanglement swapping, thus greatly speeding up the entanglement distribution.
\end{abstract}

DOI: 10.1103/PhysRevLett.114.110502

PACS numbers: 03.67.Lx, 03.67.Pp, 32.80.Qk, 42.50.Ex

Exploiting quantum systems for information processing offers many potential advantages over classical information processing like highly secure quantum networks [1-3] and powerful quantum computers [4-6]. One of the main challenges for the realization of functional quantum computers is to perform gates with sufficiently high quality so that the remaining errors can be suppressed by error correction codes, which makes the computation fault tolerant [7]. At the same time, applications to long distance quantum communication can be enabled by quantum repeaters, which combine probabilistic entanglement generation over short distances with subsequent entanglement connection steps [3]. For these protocols, the probabilistic nature of the entanglement generation is acceptable, but it is essential that high-fidelity entanglement is achieved conditioned on a heralding measurement. Experimentally, such high-fidelity entanglement is often much easier to implement and may be realized in situations where it is impossible to perform any quantum operations deterministically. Here we introduce a similar concept for gate operations and develop the concept of heralded quantum gates with integrated error detection. In the resulting gate, the infidelity, which would be present for a deterministic gate is converted into a failure probability, which is heralded by an auxiliary atom. Once successful, the resulting gate can have an arbitrarily small error. Such heralded gates could facilitate fault tolerant quantum computation since detectable errors may be easier to correct than undetectable errors [8-10]. Alternatively, it can be directly incorporated into quantum repeater architectures for long distance quantum communication.

Optical cavities are ideal for conversion between the stationary gate qubits and flying qubits (photons), which is fundamental for quantum networks [11-13]. Quantum gates can, in principle, also be directly implemented in optical cavities [14], but the experimental requirements for this are very challenging due to spontaneous emission and cavity loss. The essential parameter quantifying this is the cooperativity of the atom-cavity system, $C$. It has been argued that directly implementing gates in optical cavities leads to a poor error scaling $1-F \propto 1 / \sqrt{C}$, where $F$ is the fidelity of the gate $[15,16]$. However, as a result of the integrated error detection, the heralded gates that we propose exhibit high fidelities when successful. This enables efficient entanglement swapping and removes the necessity of intermediate entanglement purification in quantum repeaters, thus increasing the distribution rate significantly. Compared to using other deterministic, cavity based gates, an increase in the rate of up to 2 orders of magnitude can be achieved for modest cooperativities $(<100)$ and a distance of $1000 \mathrm{~km}$ [17].

The basic idea is to use a heralding auxiliary atom in addition to qubit atoms in the same cavity. One of the atomic qubit states, e.g., state $|1\rangle$ couples to the cavity mode while $|0\rangle$ is completely uncoupled [see Fig. 1(a)]. Such a system has previously been considered for twoqubit gates [16,18-21], multiqubit gates [18,22], and photon routing [23]. If any of the qubit atoms is in state $|1\rangle$, the cavity resonance is shifted compared to the bare cavity mode, which can be exploited to make a gate between two or more qubits by reflecting single photons off the cavity [18]. The efficiency of such schemes, however, is limited by photon losses, inefficient detectors and nonideal single photon sources $[21,23]$. We circumvent these problems by introducing an auxiliary atom in the cavity to serve as both an intracavity photon source and a 


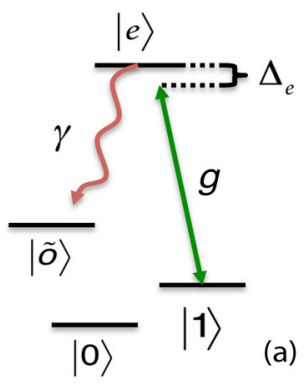

I
I
I
I
I
I
I
I
I

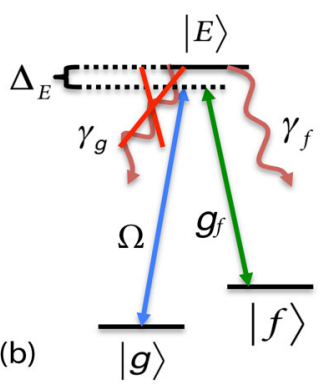

FIG. 1 (color online). (a) Level structure of the qubit atoms. Only state $|1\rangle$ couples to the cavity and we assume that the excited level decays to some level $|\tilde{o}\rangle$, possibly identical to $|f\rangle$ or $|0\rangle$. (b) Level structure of the auxiliary atom and the transitions driven by the weak laser $(\Omega)$ and the cavity $\left(g_{f}\right)$. We assume that $|E\rangle \leftrightarrow|f\rangle$ is a closed transition, i.e., $\gamma_{g}=0$.

detector. As opposed to previous heralded gates in optical cavities, which relied on the null detection of photons leaving the cavity [24-26], the final heralding measurement on the atom can then be performed very efficiently.

In our approach, the auxiliary atom has two metastable states $|g\rangle,|f\rangle$, which can be coupled through an excited state $|E\rangle$ [see Fig. 1(a)]. We assume the $|E\rangle \leftrightarrow|f\rangle$ transition to be energetically close to the cavity frequency and to be a nearly closed transition, so that we need to drive the $|g\rangle \rightarrow|E\rangle$ transition, e.g., with a two-photon process (see below). The gate can be understood through the phase evolution imposed on the atoms. We consider adiabatic excitation of the auxiliary control atom via the stimulated Raman adiabatic passage [27,28], driven by an external driving pulse with Rabi frequency $\Omega(t)$ and a coupling to the cavity photon $g_{f}$. In the case when all the qubit atoms are in the noncoupled states $|00 \ldots 0\rangle$, an adiabatic excitation will result in a dark state $\sim g_{f}|0, g\rangle-\Omega|1, f\rangle$ with zero energy and vanishing phase. Here the number refers to the number of cavity photons. However, the qubit states $\Psi$ with at least one of the qubit atoms in the coupled state, results in a cavity-induced shift of the state $|1, f, \Psi\rangle$, which in turn, causes an ac Stark shift and dynamical phase to be imprinted into the $|g, \Psi\rangle$ state after the driving pulse is turned off. All states but the completely uncoupled qubit state $|00 \ldots 0\rangle$ will thus acquire a phase, the magnitude of which depends on the length of the driving pulse. With an appropriate pulse length and simple single qubit rotations, we can use this to realize a general $N$-qubit Toffoli gate or a control-phase $(\mathrm{CZ})$ gate.

Naively, the gates will be limited by errors originating from cavity decay and spontaneous emission from the atoms, which carry away information about the qubit state. These errors are, however, detectable since the auxiliary atom will be trapped in state $|f\rangle$ if either a cavity excitation or an atomic excitation is lost. Conditioning on detecting the auxiliary atom in state $|g\rangle$ at the end of the gate thus rules out the possibility of any dissipative quantum jumps having occurred during the gate. As a result, the conditional fidelity of the gate is greatly enhanced at the modest cost of a finite but potentially low failure probability.

We now analyze the performance of the gates and derive the success probabilities, gate times, and gate errors (see Table S1 in [29]) The Hamiltonian in a proper rotating frame is (see Fig. 1)

$$
\begin{aligned}
\hat{H}= & \Delta_{E}|E\rangle\langle E|+g_{f}(\hat{a}|E\rangle\langle f|+\text { H.c. })+\hat{V}+\hat{V}^{\dagger} \\
& +\sum_{k} \Delta_{e}|e\rangle_{k}\langle e|+g\left(\hat{a}|e\rangle_{k}\langle 1|+\text { H.c. }\right),
\end{aligned}
$$

where $k$ labels the qubit atoms $(\hbar=1), 2 \hat{V}=\Omega|E\rangle\langle g|$ and we have assumed that all couplings $(g, \Omega)$ are real. We have defined $\Delta_{E}=\omega_{E}-\omega_{g}-\omega_{L}$, and $\Delta_{e}=\omega_{e}-\omega_{g}-\omega_{L}+$ $\omega_{f}-\omega_{1}$, where $\omega_{L}$ is the laser frequency and otherwise $\omega_{x}$ is the frequency associated with level $x$. We describe the cavity decay and atomic spontaneous emission with Lindblad operators so that $\hat{L}_{0}=\sqrt{\kappa} \hat{a}$ corresponds to the cavity decay, $\hat{L}_{f}=\sqrt{\gamma_{f}}|f\rangle\langle E|$ to the decay of the excited state of the auxiliary atom, and $\hat{L}_{k}=\sqrt{\gamma}|\tilde{o}\rangle_{k}\langle e|$ describes the decay of the excited qubit states to some arbitrary ground state $|\tilde{o}\rangle$. The nature of $|\tilde{o}\rangle$ is not important for the dynamics of the gates and it may or may not coincide with $|0\rangle$ or $|1\rangle$.

We assume a weak driving pulse justifying for a perturbative treatment of $\hat{V}$ using the formalism of Ref. [31]. In the perturbative description, we adiabatically eliminate the coupled excited states of the atoms and the cavity (assuming $\Omega^{2} / \Delta_{E} \ll \Delta_{E}$ and $\Omega \ll g$ ), which leads to an energy shift of the ground states but otherwise conserves them since the Hamiltonian cannot connect different unexcited states without decay. The dynamics are therefore described by an effective Hamiltonian, $\hat{H}_{\text {eff }}=|g\rangle\langle g| \sum_{n} \Delta_{n} \hat{P}_{n}$, where

$\Delta_{n}=\operatorname{Re}\left\{\frac{-\frac{\Omega^{2}}{4 \gamma}\left[\left(\frac{\Delta_{e}}{\gamma}-i / 2\right) i+2 n C\right]}{\left(2 \frac{\Delta_{e}}{\gamma}-i\right)\left[\left(2 \frac{\Delta_{E}}{\gamma}-i\right) i / 4+C\right]+\left(2 \frac{\Delta_{E}}{\gamma}-i\right) n C}\right\}$

and $\hat{P}_{n}$ projects on the states with $n$ qubits in state $|1\rangle$. For simplicity, we have assumed that the auxiliary atom is identical to the qubit atoms such that $g_{f}=g$ and $\gamma_{f}=\gamma$ (see [29] for a more general treatment) and we have defined the cooperativity $C=g^{2} / \gamma \kappa$. We consider the limit $C \gg 1$ and from Eq. (2) we find that the energy shift, in the case when all qubit atoms are in $|0\rangle$, becomes very small $\Delta_{0} \sim \Delta_{E} \Omega^{2} /\left(16 \gamma^{2} C^{2}\right) \rightarrow 0$; i.e., we drive into a zero energy dark state as mentioned in the description above. On the contrary, for $n>0$, the $C$ in the nominator of $\Delta_{n}$ reflects that the coupling of the qubit atoms shifts the cavity resonance and as a result an ac stark shift of $\sim \Omega^{2} / \Delta_{E}$ is introduced. Furthermore, we find that in the effective 
evolution, errors caused by spontaneous emission or cavity decay $\left(\hat{L}_{0}, \hat{L}_{f}, \hat{L}_{k}\right)$ project the system out of the effective space into orthogonal subspaces, which allows for an efficient error detection by measuring the ancilla atom.

The dynamics described by $\hat{H}_{\text {eff }}$ can be used to implement a Toffoli gate. Assuming the qubit atoms to be on resonance $\left(\Delta_{e}=0\right)$ and having $\Delta_{E} \sim \gamma \sqrt{C}$ gives energy shifts $\Delta_{n>0} \sim \Omega^{2} /(4 \gamma \sqrt{C})$ while $\Delta_{0} \sim \mathcal{O}\left(\Omega^{2} / C^{3 / 2}\right)$. Hence, $|00 \ldots 0\rangle$ is the only state that remains unshifted and we can choose a gate time of $t_{T} \sim 4 \pi \sqrt{C} \gamma / \Omega^{2}$ to make a Toffoli gate. By conditioning on measuring the auxiliary atom in state $|g\rangle$ at the end of the gate, the detectable errors from cavity decay and spontaneous emission only reduce the success probability instead of reducing the fidelity. Consequently, the fidelity becomes limited by more subtle, undetectable errors (see Ref. [29]). The dominant error originates from the qubit dependent decay rate $\Gamma_{n}$ of $|g\rangle \rightarrow|f\rangle$. As we demonstrate in Ref. [29], this leads to a fidelity lower bounded by $1-F \lesssim 0.3 / C$, with a success probability of $P_{\mathrm{s}} \sim 1-3 / \sqrt{C}$. This is a substantial improvement over the leading error in the case of deterministic cavity-assisted gates. For generic states, the fidelity can even be markedly higher, and improving with increasing particle number $N$ [29].

In the special case of only two qubits, the Toffoli gate is referred to as a $\mathrm{CZ}$ gate, and in this case, we can even improve the gate to have an arbitrarily small error by combining it with single qubit rotations. For the general Toffoli gate discussed above, we needed $\Delta_{e}=0$ to ensure the correct phase evolution, but making the single qubit transformations $|0\rangle \rightarrow e^{-i \Delta_{0} t / 2}|0\rangle$ and $|1\rangle \rightarrow e^{-i\left(\Delta_{1}-\Delta_{0}\right) t / 2}|0\rangle$, at the end of a driving pulse of length $t_{\mathrm{CZ}}=\mid \pi /$ $\left(\Delta_{2}-2 \Delta_{1}+\Delta_{0}\right) \mid$, ensures the right phase evolution of the $\mathrm{CZ}$ gate without any constraints on $\Delta_{e}$. Hence, it is possible to tune $\Delta_{e}$ to eliminate the detrimental effect of having a qubit dependent decay rate. Choosing $\Delta_{E}=(\gamma / 2) \sqrt{4 C+1}$ and $\Delta_{e}=\frac{1}{2} C \gamma^{2} / \Delta_{E}$ ensures $\Gamma_{0}=\Gamma_{1}=\Gamma_{2}$, and thus removes all dissipative errors from the heralded gate. The conditional error is then limited only by nonadiabatic effects, which can, in principle, be made arbitrarily small by reducing the driving strength. The success probability is $1-P_{\mathrm{s}} \sim 6 / \sqrt{C}$ in the limit $C \gg 1$ [see Fig. 2(a)]. We thus have a heralded two-qubit gate with an arbitrarily small error and a success probability that can approach 1 (it is possible to decrease the scaling factor of the probability from $\sim 6$ to $\sim 3.4$ at the expense of an error scaling as $1 / C$ by tuning $\Delta_{E}, \Delta_{e}$ ).

We now consider the gate time. The gate time of the Toffoli gate is $t_{T} \sim 4 \pi \sqrt{C} \gamma / \Omega^{2}$ and for the $C Z$ gate we have $t_{\mathrm{CZ}} \sim 15 \pi \sqrt{C} \gamma /\left(2 \Omega^{2}\right)$ for $C \gg 1$. Since $t_{\mathrm{CZ}}>t_{T}$ we focus on $t_{\mathrm{CZ}}$. The gate time is set by the strength $(\Omega)$ of the driving pulse, which is limited by nonadiabatic errors. This is investigated in the Supplemental Material where we also verify our analytical results numerically [29]. Assuming

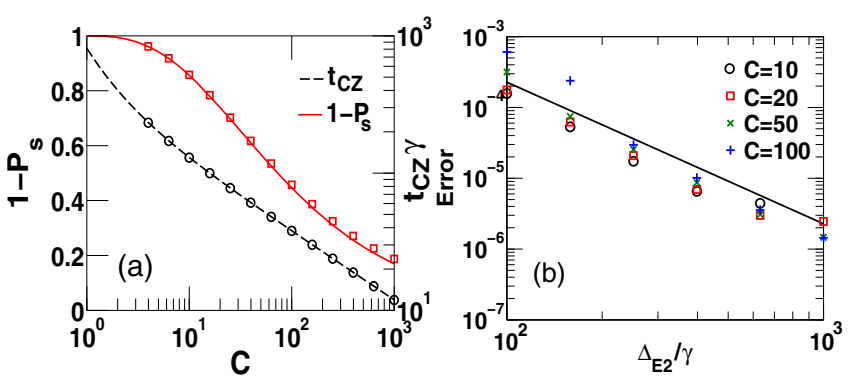

FIG. 2 (color online). (a) Failure probability $\left(1-P_{s}\right.$, left axis) and gate time ( $t_{\mathrm{CZ}}$, right axis) as a function of the cooperativity (C) for the $\mathrm{CZ}$ gate. The gate time is in units of the inverse linewidth $1 / \gamma$ of the qubit atoms. We have assumed a driving of $\Omega=\sqrt{C} \gamma / 4$. (b) Gate error as a function of the detuning $\Delta_{E 2}$ in the two-photon-driven $\mathrm{CZ}$ gate for $C=10,20,50$, and 100 . We have assumed that $\Omega_{\mathrm{MW}}=4 \gamma C^{1 / 4}$ and that $\gamma_{g}=\gamma$. The gate error decreases as $\gamma^{2} / \Delta_{E 2}^{2}$ and is independent of $C$. We have assumed $\Omega \sim \Delta_{E 2} / 8$ resulting in a gate time $\sim 400 / \gamma$. Solid (dashed) lines are analytical results and symbols are numerical simulations (see [29]). For both plots, we have assumed $\kappa=100 \gamma$.

realistic parameters of $\kappa=100 \gamma$ [23,32], we find that a driving of $\Omega=\sqrt{C} \gamma / 4$ keeps the nonadiabatic error of the gate below $4 \times 10^{-5}$ for $C \leq 1000$. The gate times decreases as $1 / \sqrt{C}$ as shown in Fig. 2(a). For a cooperativity of 100 the gate time is $\approx 1 \mu \mathrm{s}$ for typical atomic decay rates.

So far, we have assumed a model where there is no decay from $|E\rangle \rightarrow|g\rangle$. In real atoms, there will, however, always be some decay $|E\rangle \rightarrow|g\rangle$ with a decay rate $\gamma_{g}>0$. The result of such an undetectable decay is that both the $\mathrm{CZ}$ gate and the Toffoli gate will have an error $\sim \gamma_{g} /(\gamma \sqrt{C})$. To make this error small, it is thus essential to suppress the branching ratio $\gamma_{g} / \gamma$. Below we show how to suppress $\gamma_{g}$ by driving the $|g\rangle \rightarrow|E\rangle$ transition with a two-photon process. As a result, we realize a $\mathrm{CZ}$ gate with an error arbitrarily close to zero and a Toffoli gate with an error scaling as $1 / C$ even for a realistic atomic system.

Specifically we think of a level structure for the auxiliary atom, shown in Fig. 3, where we still assume $|E\rangle \leftrightarrow|f\rangle$ to be a closed transition. For simplicity, we have also assumed $\left|E_{2}\right\rangle \leftrightarrow|g\rangle$ to be a closed transition. Such a level structure could, e.g., be realized in ${ }^{87} \mathrm{Rb}$ as shown in Fig. 3. We assume that a microwave field couples the two excited states such that we can have a two-photon transition from $|g\rangle \rightarrow|E\rangle$ and that $\Omega$ is small, allowing for a perturbative treatment of the coupling. Thus we can map the system to a simple three-level atom with levels $|g\rangle,|E\rangle$, and $|f\rangle$ and a decay rate $\tilde{\gamma}_{g}$ and drive $\tilde{\Omega}$ between $|g\rangle$ and $|E\rangle$, determined by the two-photon driving process as shown in Fig. 3. The dynamics are thus similar to what we have already described for the simple three-level atom except that we have the extra decay $\tilde{\gamma}_{g}$ that introduces an error in the gates $\sim\left(\tilde{\gamma}_{g} / \gamma\right) / \sqrt{C}$, as previously described. In the limit $C \gg 1$, 


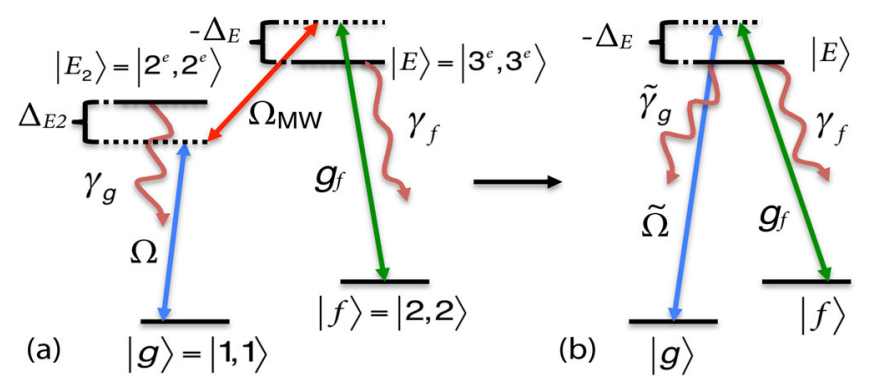

FIG. 3 (color online). (a) Level structure of the auxiliary atom and the transitions driven by a weak laser $(\Omega)$, a microwave field $\left(\Omega_{\mathrm{MW}}\right)$, and the cavity $\left(g_{f}\right)$. We assume that $|E\rangle \leftrightarrow|f\rangle$ is a closed transition and, for simplicity, we also assume that $\left|E_{2}\right\rangle \leftrightarrow|g\rangle$ is a closed transition but this is not a necessity. Here $\left|r^{(e)}, r^{(e)}\right\rangle$ with $r=1,2,3$ refers to how the atom may be realized in the $\left(5^{2} P_{3 / 2}\right)$ states $\left|F^{(e)}=r, m^{(e)}=r\right\rangle 5^{2} S_{1 / 2}$ of $\mathrm{Rb}^{87}$. (b) Effective threelevel atom realized by mapping the two-photon drive to give an effective decay rate $\tilde{\gamma}_{g}$ and an effective drive $\tilde{\Omega}$.

we find $\tilde{\gamma}_{g} / \gamma \sim\left(\gamma_{g} \Omega_{\mathrm{MW}}^{2} / 4 \gamma \Delta_{E 2}^{2}\right)$. Thus by increasing $\Delta_{E 2}$, we can, in principle, make these errors arbitrarily small. The error of the $\mathrm{CZ}$ gate for different $\Delta_{E 2}$ is shown in Fig. 2(b), assuming an initial state of $(|0\rangle+|1\rangle)^{\otimes 2}$. Note that in order to prevent an increasing scattering probability of level $|E 2\rangle$, we need to have $\Omega_{\mathrm{MW}} \propto C^{1 / 4}$ resulting in a gate error that is independent of the cooperativity [29]. The success probability and time of the gates are the same as before with $\Omega \rightarrow \tilde{\Omega} \sim\left(\Omega_{\mathrm{MW}} \Omega / 2 \Delta_{E 2}\right)$. With similar considerations about the validity of our perturbation as before, we find that for realistic parameters, we can use $\Omega=\Delta_{E 2} / 8, \Omega_{\mathrm{MW}} \sim 4 \gamma C^{1 / 4}$ resulting in a gate time of $\sim 10 \mu$ s for typical atomic decay rates and $C \lesssim 1000$ [29].

As an example implementation, we consider ultracold ${ }^{87} \mathrm{Rb}$ atoms coupled to nanophotonic cavities [23,32]. There are some additional errors originating from the extra states in the ${ }^{87} \mathrm{Rb}$ atoms in this case. In Ref. [29], we treat these errors and find that with a detuning of $\Delta_{E 2}=$ $100 \gamma$ and a cooperativity of $C \approx 100$, a heralded $C Z$ gate with $\sim 67 \%$ success probability and a heralded error of $\approx 10^{-3}$ can be realized in $\approx 10 \mu \mathrm{s}$ time. This justifies neglecting atomic decoherence which is typically much slower. Alternatively, the gate can be implemented with atomlike solid-state qubits such as nitrogen-vacancy and silicon-vacancy centers in diamond [33]. These systems can exhibit closed transitions and long-lived electronic spin states which are the essential requirement for the gate [34], while high cooperativities are possible in diamond nanocavities [35]. A particular advantage of such a system is the long-lived nuclear spin degrees of freedom, which allows each of the color centers to act as a multiqubit quantum network node [36]. By entangling electronic spins via the heralded gate, a high-fidelity, fully deterministic gate can subsequently be performed on qubits stored in nuclear spins [16].
As a particular application, we consider a quantum repeater where entanglement is first created in small segments (links), which are subsequently connected using entanglement swapping [37]. By organizing the repeater in a tree structure, the probabilistic nature of the gate can be efficiently circumvented. The success rate of distributing entanglement across the total distance $L$, scales as $\sim\left(L / L_{0}\right)^{1-\log _{2}(3 / p)}$, where $p<1$ is the success probability of the swap, $L$ is the total distribution distance and $L_{0}$ is the length between the links [17] (note that in the limit $p \rightarrow 1$, the above expression underestimates the rate, e.g., for $p=1$ the actual rate is $\sim 3$ times faster for 128 links). This is a substantial improvement over direct transmission where the success rate scales exponentially with $L$. For a realization with nuclear spin memories where the swap can be performed deterministically, the rate can scale even better as $\sim \log _{2}\left(L / L_{0}\right)^{-1}$. In order to maintain the favorable scaling without resorting to time consuming purification, the total number of links $N_{\max }$ should be kept below $N_{\max } \sim-\ln \left(F_{\text {final }}\right) /\left(\epsilon_{0}+\epsilon_{g}\right)$, where $F_{\text {final }}$ is the required fidelity of the final distributed pair and $\epsilon_{0}, \epsilon_{g} \ll 1$ are the errors of the initial entanglement generation and the entanglement swapping, respectively. Thus, it is essential that the errors are kept small, which can be obtained with the heralded gate.

In conclusion, we have introduced a heralded two-qubit quantum gate with a conditional fidelity arbitrarily close to unity and an $\mathrm{N}$-qubit Toffoli gate with an error scaling as $1 / C$. The gates have a built-in error detection process, which removes the necessity of extracting the error by the more complicated process of entanglement purification or quantum error correction. Our gate is designed for the specific case of optical cavities, and allows exploiting realistic systems for quantum communication, even though the error rate would inhibit this with deterministic gates. Similar advantages can be realized in other systems such as those based on circuit QED, where certain errors could be heralded and thus alleviate the daunting requirements of fault tolerant computation.

We thank Jeff Thompson for helpful discussions and gratefully acknowledge the support from the Lundbeck Foundation, NSF, CUA, DARPA, AFOSR MURI, and ARL. The research leading to these results has also received funding from the European Research Council under the European Union's Seventh Framework Programme (FP/ 2007-2003) through SIQS (Grant No. 600645) and ERC Grant QIOS (Grant No. 306576).

[1] J. I. Cirac, P. Zoller, H. J. Kimble, and H. Mabuchi, Phys. Rev. Lett. 78, 3221 (1997).

[2] H. J. Kimble, Nature (London) 453, 1023 (2008).

[3] L.-M. Duan, M. D. Lukin, J. I. Cirac, and P. Zoller, Nature (London) 414, 413 (2001). 
[4] T. D. Ladd, F. Jelezko, R. Laflamme, Y. Nakamura, C. Monroe, and J. L. O'Brien, Nature (London) 464, 45 (2010).

[5] P. W. Shor, SIAM J. Comput. 26, 1484 (1997).

[6] R. P. Feynman, Int. J. Theor. Phys. 21, 467 (1982).

[7] E. Knill, R. Laflamme, and G. J. Milburn, Nature (London) 409, 46 (2000).

[8] M. Grassl, T. Beth, and T. Pellizzari, Phys. Rev. A 56, 33 (1997).

[9] T. C. Ralph, A. J. F. Hayes, and A. Gilchrist, Phys. Rev. Lett. 95, 100501 (2005).

[10] M. Varnava, D. E. Browne, and T. Rudolph, Phys. Rev. Lett. 97, 120501 (2006).

[11] S. Ritter, C. Nölleke, C. Hahn, A. Reiserer, A. Neuzner, M. Uphoff, M. Mücke, E. Figueroa, J. Bochmann, and G. Rempe, Nature (London) 484, 195 (2012).

[12] P. Kómár, E. M. Kessler, M. Bishof, L. Jiang, A. S. Sørensen, J. Ye, and M. D. Lukin, Nat. Phys. 10, 582 (2014).

[13] S. Perseguers, G. J. Lapeyre Jr., D. Cavalcanti, M. Lewenstein, and A. Acín, Rep. Prog. Phys. 76, 096001 (2013).

[14] T. Pellizzari, S. A. Gardiner, J. I. Cirac, and P. Zoller, Phys. Rev. Lett. 75, 3788 (1995).

[15] M. J. Kastoryano, F. Reiter, and A. S. Sørensen, Phys. Rev. Lett. 106, 090502 (2011).

[16] A. S. Sørensen and K. Mølmer, Phys. Rev. Lett. 91, 097905 (2003).

[17] J. Borregaard, P. Kómár, E. M. Kessler, M. D. Lukin, and A. S. Sørensen (to be published).

[18] L.-M. Duan, B. Wang, and H. J. Kimble, Phys. Rev. A 72, 032333 (2005).

[19] L.-M. Duan and H. J. Kimble, Phys. Rev. Lett. 92, 127902 (2004).

[20] A. S. Sørensen and K. Mølmer, Phys. Rev. Lett. 90, 127903 (2003).

[21] A. Reiserer, N. Kalb, G. Rempe, and S. Ritter, Nature (London) 508, 237 (2014).

[22] S.-B. Zheng, Phys. Rev. A 87, 042318 (2013).

[23] T. G. Tiecke, J. D. Thompson, N. P. de Leon, L. R. Liu, Vuletić, and M. D. Lukin, Nature (London) 508, 241 (2014).
[24] J. Pachos and H. Walther, Phys. Rev. Lett. 89, 187903 (2002).

[25] A. Beige, D. Braun, B. Tregenna, and P. L. Knight, Phys. Rev. Lett. 85, 1762 (2000).

[26] V. Giovannetti, D. Vitali, P. Tombesi, and A. Ekert, Phys. Rev. A 62, 032306 (2000).

[27] S. Schiemann, A. Kuhn, S. Steuerwald, and K. Bergmann, Phys. Rev. Lett. 71, 3637 (1993).

[28] A. Kuhn, M. Hennrich, and G. Rempe, Phys. Rev. Lett. 89, 067901 (2002).

[29] See Supplemental Material at http://link.aps.org/ supplemental/10.1103/PhysRevLett.114.110502 for details of the perturbation theory, numerical simulation of the gate dynamics and discussion of additional errors, which includes Ref. [30].

[30] J. Johansson, P. Nation, and F. Nori, Comput. Phys. Commun. 184, 1234 (2013).

[31] F. Reiter and A. S. Sørensen, Phys. Rev. A 85, 032111 (2012).

[32] J. D. Thompson, T. G. Tiecke, N. P. de Leon, J. Feist, A. V. Akimov, M. Gullans, A. S. Zibrov, V. Vuletić, and M. D. Lukin, Science 340, 1202 (2013).

[33] L. Childress, R. Walsworth, and M. D. Lukin, Phys. Today 67, No. 10, 38 (2014).

[34] E. Togan, Y. Chu, A. S. Trifonov, L. Jiang, J. Maze, L. Childress, M. V. G. Dutt, A. S. Sørensen, P. R. Hemmer, A. S. Zibrov, and M. D. Lukin, Nature (London) 466, 730 (2010).

[35] M. J. Burek, Y. Chu, M. S. Z. Liddy, P. Patel, J. Rochman, D. Meesala, W. Hong, Q. Quan, M. D. Lukin, and M. Lončar, Nat. Commun. 5, 5718 (2014).

[36] P. C. Maurer, G. Kucsko, C. Latta, L. Jiang, N. Y. Yao, S. D. Bennett, F. Pastawski, D. Hunger, N. Chisholm, M. Markham, D. J. Twitchen, J. I. Cirac, and M. D. Lukin, Science 336, 1283 (2012).

[37] H.-J. Briegel, W. Dür, J. I. Cirac, and P. Zoller, Phys. Rev. Lett. 81, 5932 (1998). 